\title{
RECONFIGURAÇÃO ÓTIMA DE SISTEMAS DE DISTRIBUIÇÃO PARA MINIMIZAÇÃO DE PERDAS DE ENERGIA
}

\author{
Leonardo W. Oliveira* \\ leonardowiller@yahoo.com \\ Sandoval C. Junior* \\ sandovalddee.ufrj.br \\ Jeferson S. Costa ${ }^{\dagger}$ \\ jeferson_eng@hotmail.com
}

\author{
Edimar J. de Oliveira ${ }^{\dagger}$ \\ edimaralacee.ufje.br \\ José L. R. Pereira ${ }^{\dagger}$ \\ jluiz@ieee.org \\ Ivo C. S. Junior ${ }^{\dagger}$ \\ ivojeterra.com.br
}

${ }^{*}$ Universidade Federal do Rio de Janeiro - RJ

†Universidade Federal de Juiz de Fora - MG

\section{RESUMO}

Este artigo apresenta um algoritmo para a reconfiguração ótima de Sistemas de Distribuição de Energia Elétrica (SDE), com o objetivo de minimizar a perda total de energia considerando diferentes níveis de carregamento. Trata-se de um problema de programação não linear inteira mista onde a variável discreta é modelada como uma função contínua. Como consequiência, o problema proposto é resolvido através de um algoritmo passo a passo, onde em cada passo é utilizado o Método Primal-Dual de Pontos Interiores. Os multiplicadores de Lagrange são utilizados para compor o índice de sensibilidade no processo de reconfiguração. O algoritmo proposto é testado em três sistemas encontrados na literatura.

PALAVRAS-CHAVE: Perdas de Energia, Reconfiguração, Níveis de Carregamento, Algoritmo Passo a Passo, Pontos Interiores.

\section{ABSTRACT}

This paper presents an algorithm for reconfiguration of Electrical Distribution Systems in order to minimize energy losses considering different load levels. This problem is con-

Artigo submetido em 22/09/2008 (Id.: 00898)

Revisado em 15/12/2008, 05/01/2009

Aceito sob recomendação do Editor Associado Prof. Eduardo N. Asada sidered as mixed integer non linear programming, in which a continuous function is used to handle the discrete variables. As consequence, the proposed model is solved using a step by step approach where the primal-dual interior point technique is solved at each step. The Lagrange multiplies are used to evaluate the sensitivity index for the reconfiguration process. The developed algorithm is tested in three systems of the literature.

KEYWORDS: Energy Losses, Reconfiguration, Load Level, Step by Step approach, Interior Points.

\section{INTRODUÇÃO}

O problema de reconfiguração de SDE consiste na determinação de uma topologia radial e conexa da rede, através da definição dos estados (aberto ou fechado) das chaves manobráveis acopladas aos circuitos de distribuição (Sarfi et. al., 1994). Esta topologia otimiza um determinado índice de desempenho do sistema, como a redução das perdas técnicas, atendendo as restrições operacionais (Kalantar et al., 2006). Os dispositivos de chaveamento incluem: $(i)$ chaves de seccionamento ou normalmente fechadas (NF); (ii) chaves de interconexão ou normalmente abertas (NA).

Esta opção conduz a um problema de programação não linear inteira mista, de natureza combinatória devido aos es- 
tados das chaves manobráveis. Portanto, considerando-se as dimensões dos SDE de médio e grande porte, a busca por soluções ótimas é bastante complexa, pois requer a avaliação de um número elevado de alternativas. Outro aspecto que introduz mais complexidade ao problema consiste na característica radial e conexa da rede de distribuição.

Com este propósito, podem ser encontradas na literatura metodologias que utilizam técnicas de otimização discreta como Branch and Bound (Merlin and Back, 1975). No entanto, devido à natureza combinatória do problema de reconfiguração, este método requer a análise de um grande número de configurações para sistemas reais (Liu et. al., 1989; Radha et. al., 2003). Algoritmos baseados em meta-heurísticas como Simulated Annealing (Chiang and Jean-Jumeau, 1990), Colônia de Formigas (Khoa and Phan, 2006) e Busca Tabu (Mori and Ogita, 2000) também têm sido explorados para a solução deste problema, bem como algoritmos baseados em sistemas inteligentes, como os Algoritmos Genéticos (Nara et. al., 1992), as Redes Neurais Artificiais (Salazar et. al., 2006) e os que utilizam lógica fuzzy (Das, 2006).

Diversos métodos heurísticos têm sido propostos como técnica de solução para o problema de reconfiguração de SDE visando minimização de perdas (Shirmohammadi and Hong, 1989; Goswami and Basu, 1992; Peponis and Papadopoulos, 1995; Kagan, N. and Oliveira, C. C. B., 1998; McDermott et. al., 1999). Civanlar et. al. (1988), Baran and Wu (1989) e Shin et. al. (2007) utilizaram a técnica heurística denominada Branch-Exchange para a determinação de configurações com perdas de potência ativa mínimas. Estes trabalhos apresentam estratégias simplificadas para o cálculo das perdas e redução do espaço de busca, visando reduzir esforços computacionais. Mantovani et. al. (2000) propuseram um algoritmo heurístico para a minimização de perdas de potência ativa e o balanceamento de carga. O método proposto baseia-se no limite máximo de queda de tensão nos alimentadores. Gomes et. al. (2005a) apresentaram um algoritmo tendo como base uma estratégia heurística que realiza a abertura seqüencial de chaves manobráveis, a partir de uma configuração em malha, visando o menor acréscimo de perdas no SDE. Este método requer o cálculo de fluxo de carga não linear para cada abertura de chave. Em continuidade a este trabalho, Gomes et. al. (2005b) propuseram a utilização de uma outra estratégia baseada em Fluxo de Potência Ótimo (FPO), a fim de reduzir o número de simulações de fluxo de carga e, conseqüentemente, o esforço computacional.

Devido a dimensão deste problema e o tempo computacional envolvido, poucos trabalhos sobre reconfiguração consideram mais de um período de carregamento do sistema (Chen and Cho, 1993; Taleski and Rajicic, 1997; Dumbrava et. al., 2001; Venkatesh and Ranjan, 2006). A maioria dos trabalhos considera apenas um período de carregamento, onde a solução encontrada é utilizada durante as outras horas do dia. Portanto, verifica-se que o sistema não se encontra em uma configuração de rede ótima para todos os níveis de carregamento. Seguindo esta linha de pesquisa, o presente trabalho apresenta um algoritmo para a reconfiguração ótima de sistemas de distribuição considerando-se diferentes níveis de carregamento. Na metodologia proposta as chaves manobráveis são modeladas por funções contínuas e os diferentes níveis de carga são incorporados em um único problema de fluxo de potência ótimo (Silva Junior et. al., 2008). O algoritmo considera uma topologia inicial em malha e efetua a abertura sequencial de chaves, até que uma configuração radial seja obtida. Cada abertura é baseada na sensibilidade das variáveis discretas de chaveamento, obtida pelos multiplicadores de Lagrange. Para solução do FPO em cada passo do algorítimo utiliza-se o Método Primal-Dual de Pontos Interiores (MPI) (Karmakar, 1991; Granville et. al., 1996). Os resultados obtidos são comparados para avaliar a eficiência do método proposto.

\section{METODOLOGIA PROPOSTA}

A modelagem das chaves no FPO implica no tratamento de variáveis discretas que podem assumir somente dois valores: 0 (chave aberta) ou 1 (chave fechada). Portanto, uma chave pode ser representada pela função degrau unitário. No entanto, a função degrau apresenta uma descontinuidade no ponto de mudança de estado. Neste ponto, esta função não é diferenciável e, portanto, não pode ser modelada no FPO. Como alternativa, no presente trabalho é utilizada a função sigmóide (Haykin, 2002), dada pela Equação (1) e ilustrada na Figura 1.

$$
C H_{k m}\left(x_{k m}\right)=\frac{e^{\alpha \cdot x_{k m}}-1}{e^{\alpha \cdot x_{k m}}+1} \quad x_{k m} \geq 0
$$

Onde:

$x_{k m}$ Representa a variável de otimização associada à chave manobrável acoplada ao circuito $k-m$;

$C H_{k m}\left(x_{k m}\right)$ Representa o valor de posição da chave manobrável do circuito $k-m$.

A função sigmóide utilizada possui o parâmetro $\alpha=1$ conforme foi adotado em (Silva Junior et. al., 2008; Oliveira et. al., 2005). A Figura 1 mostra que o argumento da função sigmóide $\left(x_{k m}\right)$ varia em uma faixa de zero a 20. Destaca-se que o limite superior desta variável pode ser definido como qualquer valor que torna $\mathrm{CH}_{k m}\left(x_{k m}\right)$ o mais próximo possível de 1 . 


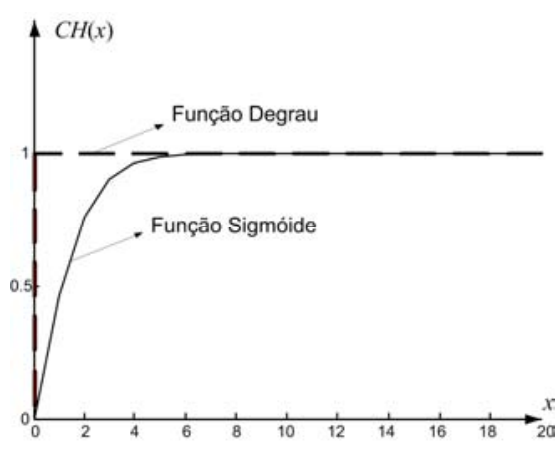

Figura 1: Função contínua de chaveamento.

O problema de FPO proposto para reconfiguração ótima de SDE é similar ao modelo proposto em (Silva Junior et. al., 2008) e pode ser formulado como:

$$
\begin{aligned}
& \min F O B= \\
& =\sum_{u=1}^{N T}\left[\sum_{k=1}^{N B}\left[\sum_{m \in \Omega k}\left[C H_{k m}\left(x_{k m}\right) \cdot\left(c e_{u} \cdot T_{u} \cdot L_{k m, u}\right)\right]\right]\right.
\end{aligned}
$$

$\mathrm{sa} /$

$$
\begin{gathered}
P g_{k, u}-P l_{k, u}+\sum_{m \in \Omega k} C H_{k m}\left(x_{k m}\right) \cdot P_{k m, u}=0 \\
Q g_{k, u}-Q l_{k, u}+\sum_{m \in \Omega k} C H_{k m}\left(x_{k m}\right) \cdot Q_{k m, u}=0 \\
L_{k m, u}=g_{k m} \cdot\left[V_{k, u}^{2}+V_{m, u}^{2}-2 \cdot V_{k, u} \cdot V_{m, u} \cdot \cos \left(\theta_{k m, u}\right)\right] \\
0 \leq x_{k m} \leq 20 \\
\bar{Z}^{\min } \leq \overline{Z_{u}} \leq \bar{Z}^{\max }
\end{gathered}
$$

Onde:

\section{$F O B$ Representa a função objetivo;}

$u$ Representa um nível de carregamento;

NT Representa o número de níveis de carregamento considerados;

$N B$ Representa o número de barras do sistema;

$\Omega k$ Representa o conjunto de barras conectadas à barra $k$; $c e_{u}$ Representa a tarifa de energia (US\$/kWh) para o nível de carregamento $u$;

$T_{u}$ Representa o intervalo de tempo que o SDE opera com o nível de carregamento $u$;

$L_{k m, u}$ Representa a perda de potência ativa no circuito $k-$ $m$ no nível de carregamento $u$;

$P g_{k, u}$ Representa a geração de potência ativa na barra $k$ no nível de carregamento $u$;

$P l_{k, u}$ Representa a carga ativa na barra $k$ no nível de carregamento $u$;

$P_{k m, u}$ Representa o fluxo de potência ativa no circuito $k-m$ no nível de carregamento $u$;

$\lambda p_{k, u}$ Representa o multiplicador de Lagrange associado à equação de balanço de potência ativa (2.1) no nível de carregamento $u$;

$Q g_{k, u}$ Representa a geração de potência reativa na barra $k$ no nível de carregamento $u$;

$Q l_{k, u}$ Representa a carga reativa na barra $k$ no nível de carregamento $u$;

$Q_{k m, u}$ Representa o fluxo de potência reativa no circuito $k-$ $m$ no nível de carregamento $u$;

$\lambda q_{k, u}$ Representa o multiplicador de Lagrange associado à equação de balanço de potência reativa (2.2) no nível de carregamento $u$;

$g_{k m}$ Representa a condutância do circuito $k-m$;

$V_{k, u}$ Representa a magnitude de tensão da barra $k$ no nível de carregamento $u$;

$\theta_{k m, u}$ Representa a defasagem angular entre as barras $k$ e $m$ no nível de carregamento $u$;

$\overline{Z_{u}}$ Representa o vetor das demais variáveis do FPO para o nível de carregamento $u$;

$\bar{Z}^{\text {min }}, \bar{Z}^{\text {max }}$ Representam os limites mínimo e máximo das variáveis $\overline{Z_{u}}$, respectivamente.

A Equação (2) define a função objetivo do FPO, e está associada à minimização do custo da perda total de energia no sistema em todos os níveis de carga considerados. Caso o circuito $k-m$ não possua chave manobrável, $C H_{k m}\left(x_{k m}\right)$ é fixada no valor 1 .

As Equações (2.1) e (2.2) correspondem às restrições de balanço de potência ativa e reativa, respectivamente. $\mathrm{O}$ valor de posição $C H_{k m}\left(x_{k m}\right)$ é multiplicado pelas expressões dos fluxos de potência ativa $P_{k m, u}$ e reativa $Q_{k m, u}$. 
A Equação (2.3) é utilizada para cálculo da perda de potência ativa no circuito $k-m$. A Equação (2.4) define os limites da variável associada à chave do circuito $k-m \quad\left(x_{k m}\right)$. As demais variáveis de otimização possuem os limites estabelecidos em (2.5). Neste conjunto de restrições, incluem-se os limites de tensão nodal e geração ativa.

O problema de FPO em (2) é solucionado utilizando-se o Método Primal-Dual de Pontos Interiores (MPI) (Granville et. al., 1996). Após a solução deste problema, os valores de posição das chaves manobráveis $\left(\mathrm{CH}_{\mathrm{km}}\right)$ encontram-se no intervalo contínuo $[0,1]$. Porém, na prática, os estados das chaves são discretos, ou seja, ligado $\left(\mathrm{CH}_{\mathrm{km}}=1\right)$ ou desligado $\left(C H_{k m}=0\right)$. Logo, a obtenção de uma solução prática para este problema requer uma estratégia para definição dos estados das chaves. Esta estratégia baseia-se no cálculo de um índice de sensibilidade, apresentado a seguir.

\section{3 ÍNDICE DE SENSIBILIDADE PRO- POSTO}

A metodologia de reconfiguração ótima proposta no presente trabalho visa determinar, a partir da configuração em malha, o número mínimo de chaves manobráveis cuja abertura resulta em uma operação radial e com o mínimo acréscimo de perdas. Baseado nesta premissa, o índice de sensibilidade proposto para determinação do estado das chaves corresponde a uma medida do impacto que a abertura de um determinado circuito causa na função objetivo do FPO. Este índice é dado por:

$$
S S_{k m}=\left|C H_{k m}\left(x_{k m}\right) \cdot\left(\lambda p_{k}-\lambda p_{m}\right) \cdot \frac{N_{k m}}{\left(V_{k}-V_{m}\right)}\right|
$$

Onde:

$S S_{k m}$ Representa o índice de sensibilidade para definição do estado da chave $k-m$;

$N_{k m}$ Representa o fluxo de potência aparente no circuito $k-$ $m$.

Na Equação (3), o índice proposto $\left(S S_{k m}\right)$ é proporcional ao valor de posição $C H_{k m}\left(x_{k m}\right)$. Quanto menor o valor de $C H_{k m}\left(x_{k m}\right)$, maior é a tendência de abertura do circuito $k-m$ para a minimização de perdas, estabelecida pela função objetivo.

A diferença $\left(\lambda p_{k}-\lambda p_{m}\right)$ corresponde ao impacto na função objetivo em (2) devido a uma variação de fluxo no circuito $k-m$. Em outras palavras, quanto menor $\left(\lambda p_{k}-\lambda p_{m}\right)$, menor é o aumento das perdas no SDE devido à abertura do circuito $k-m$.

O índice de decisão $S S_{k m}$ também é função do fluxo da potência aparente $N_{k m}$, pois a abertura de uma determinada chave resulta em uma redistribuição de fluxos por toda a rede de distribuição. A abertura do circuito com menor $N_{k m}$ causa menos alterações na rede, em conseqüência, mínimo afastamento do ponto de operação obtido com a rede em malha.

Adicionalmente, o índice $S S_{k m}$ é inversamente proporcional à queda de tensão no circuito $k-m \quad\left(V_{k}-V_{m}\right)$. Isto retrata a condição de que o circuito que apresenta grande valor de queda de tensão deve ser evitado para conduzir potência e, portanto, terá preferência para abertura (Lin and Chin, 1998).

Considerando os aspectos abordados anteriormente, concluise que a chave preferencial para abertura é aquela que possui o menor índice $S S_{k m}$, pois sua abertura resulta no menor acréscimo de perdas em relação à configuração em malha. Para que o índice de sensibilidade $S S$ retrate com fidelidade a tendência de abertura de chaves em direção ao ponto de mínimas perdas, as chaves devem ser abertas sequencialmente. Para tanto, um algoritmo de reconfiguração ótima de SDE é proposto no presente trabalho, o qual é apresentado a seguir.

\section{ALGORITMO PROPOSTO}

O Algoritmo de Reconfiguração de Sistemas de Distribuição (ARSD) é apresentado pelo fluxograma da Figura 2.

No ARSD considera-se a rede inicialmente configurada em malha através do fechamento de todas as chaves de interconexão (NA). A partir desta configuração, as chaves são abertas em seqüência até que uma configuração radial seja obtida. As etapas deste algoritmo são descritas a seguir.

\section{Etapa-1}

A Etapa-1 do ARSD tem como objetivo determinar uma Lista de Chaves Manobráveis do sistema (LCM), (Gomes et. al., 2005a). Esta lista pode ser previamente obtida dos dados da rede elétrica, ou determinada por um procedimento que identifica a formação de ilhamento devido à abertura de circuito. Neste procedimento, a LCM é inicialmente composta de todas as chaves. A partir daí, efetua-se um processo de busca no grafo da rede de distribuição pelos nós conectados através de uma única ligação.

\section{Etapa-2}

O objetivo desta etapa é determinar os valores das variáveis necessários para o cálculo do índice de sensibilidade $S S$, formulado na Equação (3), visando a definição da próxima 


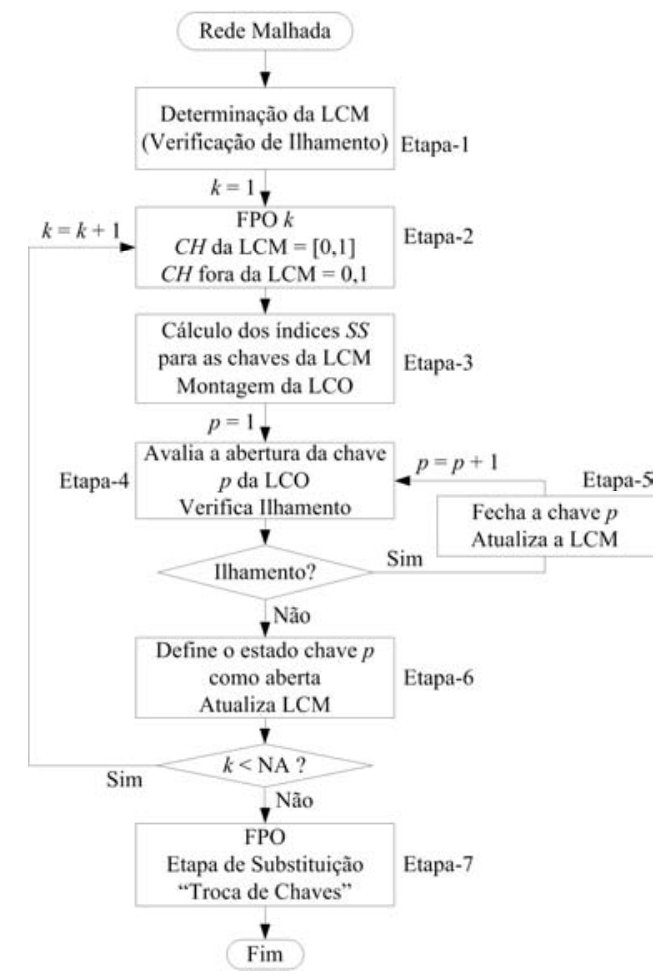

Figura 2: Fluxograma do algoritmo proposto (ARSD).

chave para abertura (chave $k$ ). Para tanto, efetua-se a resolução do FPO (problema 2) considerando-se as variáveis associadas às chaves da LCM. Os valores de $x$ para as chaves que não se encontram na LCM são fixados de acordo com os estados das mesmas nos seguintes valores: $x=0$ para as chaves abertas, pois neste caso $C H(x)=0$ como se pode observar da Figura $1 ; x=20$ para as chaves fechadas, pois neste caso $C H(x)=1$. Portanto, após a resolução do FPO, os valores das variáveis $x$ associadas às chaves da LCM encontram-se no intervalo contínuo [0,1], enquanto que as variáveis associadas às chaves que não pertencem à LCM assumem o valor 0 ou o valor 1 .

\section{Etapa-3}

Nesta etapa, é efetuado o cálculo do índice de sensibilidade SS para as chaves da LCM, conforme Equação (3). As variáveis necessárias para este cálculo são obtidas da solução do FPO (2). As chaves prioritárias para abertura são listadas de acordo com a ordem crescente dos índices SS. Esta lista foi denominada LCO (Lista de Chaves Ótimas). Ou seja, a primeira chave da LCO é a que possui o menor índice $S S$ e que, portanto, deve ser aberta.

\section{Etapa-4}

Esta etapa consiste na avaliação da abertura da próxima chave (chave $p$ ) da LCO, partindo da primeira chave, associada ao menor índice de sensibilidade SS. Esta avaliação consiste numa verificação no grafo da rede elétrica da possibilidade de ocorrência de ilhamento devido à abertura da chave $p$. Destaca-se que nesta etapa não é necessária resolução de FPO, pois a verificação de ilhamento é realizada via processo de busca no grafo da rede descrito na Etapa-1.

\section{Etapa-5}

Esta etapa é executada no caso de formação de ilhamento após a abertura da chave $p$. Neste caso, a chave $p$ é fechada e é retirada da LCM. Na sequiência, a Etapa-4 é novamente executada para abertura e avaliação da próxima chave $(p=p$ +1 ) da LCO.

\section{Etapa-6}

Caso não ocorra ilhamento, a chave $p$ é definida como aberta. Esta chave é, portanto, retirada da LCM uma vez que seu estado está determinado. As chaves que estão em série com a chave $p$ também são retiradas da LCM, pois neste caso sua abertura geraria ilhamento. Após a Etapa-6, o número de chaves abertas $(k)$ é comparado com o número de chaves de interconexão do sistema original (NA). Se o primeiro número é menor que o segundo, ainda existem laços na rede. Neste caso, o contador de chaves abertas $k$ é incrementado e o processo é repetido a partir da Etapa-2 para a determinação da próxima chave. Caso contrário, uma configuração radial foi obtida e o algoritmo segue para a Etapa-7.

\section{Etapa-7}

A Etapa-7 consiste em uma avaliação da possibilidade de melhoria da configuração radial obtida através de substituições ("Branch Exchange"), baseado em Civanlar et. al. (1988). Esta avaliação baseia-se em um processo combinado de fechamento da chave aberta e abertura de uma chave próxima da primeira ("chave vizinha"), a partir da configuração radial. Este processo garante a geração de novas configurações radiais. O conjunto de "chaves vizinhas" é definido através de um processo de determinação do caminho mínimo (Goldbarg and Luna, 2005), composto pelos circuitos que interligam os dois terminais de uma determinada chave aberta.

Após cada substituição, é calculada a perda total de energia para a nova configuração gerada via FPO. Se esta perda é inferior àquela calculada para a configuração anterior, esta é substituída pela nova configuração. Este processo é realizado para cada chave aberta, e para 1 "chave vizinha" adjacente, a partir de cada extremidade da primeira. Portanto, são realizadas 2 substituições para cada chave aberta. Nesta etapa, os estados de todas as chaves são definidos, ou seja, as variáveis $x$ associadas a estes dispositivos de chaveamento são fixadas de acordo com o estado das respectivas chaves. 


\section{MODELAGEM DOS NÍVEIS DE CARGA}

O problema de FPO proposto em (2) permite a modelagem de diferentes níveis de carregamento do sistema. Para representar três níveis de carga, por exemplo, é necessário estabelecer os seguintes vetores de variáveis de otimização: $\overline{Z_{L}}$ (carga leve), $\overline{Z_{M}}$ (carga média) e $\overline{Z_{P}}$ (carga pesada). Cada um destes conjuntos deve ser acomodado na matriz hessiana e no vetor gradiente, como mostra a Equação (4).

$$
\begin{aligned}
& {\left[\begin{array}{cccc}
W_{\bar{x}, \bar{x}} & W_{\bar{x}, \overline{Z_{L}}} & W_{\bar{x}, \overline{Z_{M}}} & W_{\bar{x}, \overline{Z_{P}}} \\
W_{\bar{x}, \overline{Z_{L}}} & W_{\overline{Z_{L}}, \overline{Z_{L}}} & & \\
W_{\bar{x}, \overline{Z_{M}}} & & W_{\overline{Z_{M}}, \overline{Z_{M}}} & \\
W_{\bar{x}, \overline{Z_{P}}} & & W_{\overline{Z_{P}}, \overline{Z_{P}}}
\end{array}\right] \cdot\left[\begin{array}{c}
\Delta_{\bar{x}} \\
\Delta \overline{Z_{L}} \\
\Delta \overline{Z_{M}} \\
\Delta \overline{Z_{P}}
\end{array}\right]=} \\
& =\left[\begin{array}{c}
G_{\bar{x}} \\
G_{\overline{Z_{L}}} \\
G_{\overline{Z_{M}}} \\
G_{\overline{Z_{P}}}
\end{array}\right]
\end{aligned}
$$

Onde:

$W_{\bar{x}, \bar{x}}$ Representa a sub-matriz hessiana diagonal para as variáveis associadas às chaves $(x)$;

$W_{\overline{Z_{u}}}, \overline{Z_{u}}$ Representam as sub-matrizes hessiana para os diferentes níveis de carregamento;

$W_{\bar{x}, \overline{Z_{u}}}$ Representam as sub-matrizes hessiana para acoplamento entre os diferentes níveis de carregamento e as variáveis associadas às chaves $(x)$;

$\Delta_{\bar{x}}$ Representa o vetor independente para as variáveis associadas às chaves;

$\Delta_{\overline{Z_{u}}}$ Representam os vetores independentes para os diferentes níveis de carregamento;

$G_{\bar{x}}$ Representa o vetor gradiente para as variáveis associadas às chaves;

$G_{\overline{Z_{u}}}$ Representam os vetores gradiente para os diferentes níveis de carregamento.

Destaca-se que para todos os níveis, uma mesma chave está associada a uma única variável $x$. Portanto, estes níveis estão acoplados entre si através do vetor $\bar{x}$.

\section{RESULTADOS}

Nesta seção, são apresentados os resultados obtidos através da aplicação do algoritmo proposto (ARSD). Para tanto, são

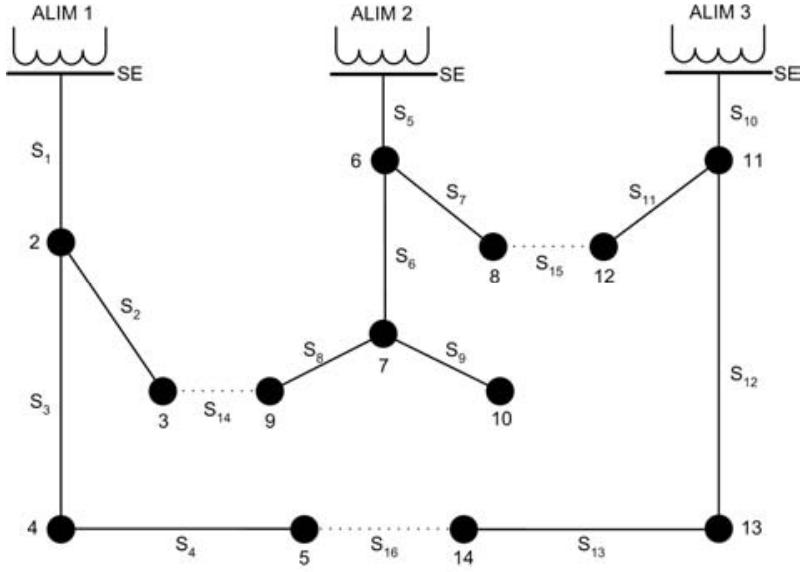

Figura 3: Sistema teste de 16 barras.

utilizados três sistemas da literatura: o sistema de 16 barras (Civanlar et. al., 1988), o sistema de 33 barras (Baran and $\mathrm{Wu}, 1989$ ) e o sistema de 83 barras (Chiou et. al., 2005). Nestes casos, a tensão da subestação é considerada igual a 1,0 p.u., e os limites de tensão nodal adotados são 0,90 p.u. e 1,01 p.u.. Os testes foram realizados utilizando-se um microcomputador AMD Athlon(tm) 64 Processor 3000+, velocidade $2 \mathrm{GHz}$, e o software MATLAB ${ }^{\circledR}$ versão 7.6.0 (R2008a).

\section{1) Sistema teste de 16 Barras}

O sistema teste de 16 barras (Civanlar et al., 1988) é composto de 3 alimentadores de $23 \mathrm{kV}$ contendo 16 circuitos. A Figura 3 apresenta o diagrama deste sistema.

Nesta topologia inicial, os circuitos representados por linhas contínuas estão acoplados a chaves normalmente fechadas (NF) e os circuitos tracejados representam as chaves normalmente abertas (NA). Nesta configuração, a perda total de potência ativa é de 511,44 kW. A seguir, será mostrada a solução passo a passo de cada etapa do algoritmo proposto para reconfiguração (ARSD), considerando-se somente um nível de carga média ( 1,0 p.u.) e minimização de perdas de potência.

\section{Etapa-1:}

O objetivo da Etapa-1 é a determinação da Lista de Chaves Manobráveis (LCM). Como os dados do sistema de 16 barras não incluem esta lista, a princípio todas as 16 chaves são consideradas manobráveis. No entanto, o processo de varredura da rede para verificação de ilhamento identifica que a chave $S_{9}$ não pode ser aberta, pois neste caso a barra 10 seria isolada, como se pode observar na Figura 3. Portanto, a chave $S_{9}$ não pode constar na LCM, apresentada a seguir:

$\mathrm{LCM}=\left\{\mathrm{S}_{1}, \mathrm{~S}_{2}, \mathrm{~S}_{3}, \mathrm{~S}_{4}, \mathrm{~S}_{5}, \mathrm{~S}_{6}, \mathrm{~S}_{7}, \mathrm{~S}_{8}, \mathrm{~S}_{10}, \mathrm{~S}_{11}, \mathrm{~S}_{12}, \mathrm{~S}_{13}\right.$, 
$\left.\mathrm{S}_{14}, \mathrm{~S}_{15}, \mathrm{~S}_{16}\right\}$.

Etapa-2- $1^{\circ}$ passo:

A Etapa-2 tem como objetivo otimizar a operação do sistema através da resolução do Fluxo de Potência Ótimo (FPO) formulado em (2). No $1^{o}$ passo, esta etapa (FPO-1) considera a rede configurada em malha, e a lista inicial LCM.

Etapa-3- $1^{\circ}$ passo:

Esta etapa efetua o cálculo dos índices de sensibilidade $S S$, conforme Equação (3), para todas as chaves da LCM. Estes índices são apresentados na Tabela 1.

Tabela 1: Índices SS, FPO-1, sistema 16 barras.

\begin{tabular}{|c|c|c|c|c|c|}
\hline Chave & $\begin{array}{c}S S \\
\left(\cdot 10^{6}\right)\end{array}$ & Chave & $\begin{array}{c}S S \\
\left(\cdot 10^{6}\right)\end{array}$ & Chave & $\begin{array}{c}S S \\
\left(\cdot 10^{6}\right)\end{array}$ \\
\hline $\mathrm{S}_{1}$ & 9,5054 & $\mathrm{~S}_{6}$ & 8,3398 & $\mathrm{~S}_{12}$ & 3,4212 \\
\hline $\mathrm{S}_{2}$ & 6,2298 & $\mathrm{~S}_{7}$ & 0,4911 & $\mathrm{~S}_{13}$ & 3,6427 \\
\hline $\mathrm{S}_{3}$ & 2,5979 & $\mathrm{~S}_{8}$ & 2,3238 & $\mathrm{~S}_{14}$ & 3,4496 \\
\hline $\mathrm{S}_{4}$ & 0,8357 & $\mathrm{~S}_{10}$ & 6,6628 & $\mathrm{~S}_{15}$ & 1,2646 \\
\hline $\mathrm{S}_{5}$ & 9,8568 & $\mathrm{~S}_{11}$ & 2,6531 & $\mathrm{~S}_{16}$ & 0,2964 \\
\hline
\end{tabular}

Após o cálculo dos índices de sensibilidade $S S$, é montada a Lista de Chaves Ótimas (LCO) para abertura contendo as chaves dispostas na ordem crescente dos respectivos índices. A Tabela 2 apresenta a LCO-1, montada na Etapa-3 deste $1^{\circ}$ passo.

Tabela 2: LCO-1, sistema 16 barras.

\begin{tabular}{|c|c|}
\hline \multirow{3}{*}{ Chaves } & $\mathbf{S}_{16}-\mathrm{S}_{7}-\mathrm{S}_{4}-\mathrm{S}_{15}-\mathrm{S}_{8}-\mathrm{S}_{3}-\mathrm{S}_{11}-\mathrm{S}_{12}-\mathrm{S}_{14}-$ \\
& $\mathrm{S}_{13}-\mathrm{S}_{2}-\mathrm{S}_{10}-\mathrm{S}_{6}-\mathrm{S}_{1}-\mathrm{S}_{5}$ \\
\hline
\end{tabular}

Observa-se que a chave $S_{16}$ está associada ao menor índice $S S$ do sistema. Logo, esta é a primeira chave da LCO-1 avaliada para abertura.

\section{Etapa-4- $1^{\circ}$ passo:}

No $1^{\circ}$ passo, a Etapa-4 tem como objetivo efetuar a avaliação da abertura de cada chave da LCO-1, partindo da primeira chave, até que não seja verificada a presença de ilhamento. Neste caso, a abertura da chave $S_{16}$ não forma ilhamento, encerrando a Etapa-4. Como não ocorreu ilhamento, a Etapa5 não será executada.

Etapa-6- $1^{\circ}$ passo:

Tendo em vista que a abertura da chave $\mathrm{S}_{16}$ não forma ilhamento na rede de distribuição, esta chave é aberta, e retirada da LCM. Da Figura 3, verifica-se que a chave $\mathrm{S}_{16}$ está em série com $\mathrm{S}_{3}$ e $\mathrm{S}_{4}$ à sua esquerda, e com $\mathrm{S}_{12}$ e $\mathrm{S}_{13}$ à sua direita. Ou seja, após a abertura de $S_{16}$, a abertura de $S_{3}, S_{4}, S_{12}$ ou $\mathrm{S}_{13}$ não é permitida, pois formaria ilhamento na rede. Logo, estas chaves também devem ser retiradas da LCM, que deve ser atualizada para a LCM-1 mostrada a seguir:

LCM-1 $=\left\{\mathrm{S}_{1}, \mathrm{~S}_{2}, \mathrm{~S}_{5}, \mathrm{~S}_{6}, \mathrm{~S}_{7}, \mathrm{~S}_{8}, \mathrm{~S}_{10}, \mathrm{~S}_{11}, \mathrm{~S}_{14}, \mathrm{~S}_{15}\right\}$.

No fim do $1^{o}$ passo ( $k=1$ ), uma única chave foi aberta $\left(\mathrm{S}_{16}\right)$. O número de chaves abertas é, portanto, inferior ao número de chaves de interconexão ( $\mathrm{NA}=3$ ). Logo, o contador de chaves abertas $k$ é incrementado e o algoritmo é novamente executado a partir da Etapa-2, com o objetivo de determinar a segunda chave para abertura, considerando a nova lista LCM-1.

Portanto, a segunda resolução do FPO formulado em (2) considera a rede parcialmente malhada, pois as malhas formadas a partir de $\mathrm{S}_{16}$ foram desfeitas após o $1^{\circ}$ passo. Após este cálculo, a chave $\mathrm{S}_{7}$ apresentou o menor índice $S S$, como mostra a LCO-2 apresentada na Tabela 3.

Tabela 3: LCO-2, sistema 16 barras.

\begin{tabular}{c|c} 
Chaves & $\mathbf{S}_{7}-\mathrm{S}_{15}-\mathrm{S}_{8}-\mathrm{S}_{11}-\mathrm{S}_{14}-\mathrm{S}_{2}-\mathrm{S}_{10}-\mathrm{S}_{6}-\mathrm{S}_{1}-$ \\
$\mathrm{S}_{5}$ \\
\hline
\end{tabular}

A abertura da primeira chave da $\mathrm{LCO}-2\left(\mathrm{~S}_{7}\right)$ não forma ilhamento no sistema de 16 barras da Figura 3. Portanto, esta chave é aberta, e retirada da LCM-1. A chave $\mathrm{S}_{7}$ está em série com $S_{11}$ e $S_{15}$, como mostra a figura. Logo, estas chaves também são retiradas da LCM-1, que é atualizada para LCM-2:

LCM-2 $=\left\{S_{1}, S_{2}, S_{5}, S_{6}, S_{8}, S_{10}, S_{14}\right\}$.

No fim do $2^{\circ}$ passo $(k=2)$, duas chaves foram abertas $\left(\mathrm{S}_{16}\right.$ e $S_{7}$ ). Este número ainda é inferior ao número de chaves de interconexão ( NA = 3 ). Logo, o contador de chaves abertas $k$ é novamente incrementado ( $k=3$ ) e o algoritmo da Figura 2 segue para um novo passo, a partir da Etapa-2.

O objetivo da terceira resolução de FPO (FPO-3) é determinar a próxima chave para abertura, considerando a lista de chaves manobráveis LCM-2. A Tabela 4 apresenta a LCO-3, montada neste $3^{\circ}$ passo do algoritmo proposto (ARSD).

Tabela 4: LCO-3, sistema 16 barras.

Chaves $\quad \mathbf{S}_{8}-\mathrm{S}_{14}-\mathrm{S}_{10}-\mathrm{S}_{2}-\mathrm{S}_{6}-\mathrm{S}_{1}-\mathrm{S}_{5}$

Novamente, a abertura da primeira chave da LCO-3 $\left(\mathrm{S}_{8}\right)$ não forma ilhamento, e então esta operação é efetuada. No fim do 
$3^{\circ}$ passo, três chaves foram abertas $\left(\mathrm{S}_{16}, \mathrm{~S}_{7}\right.$ e $\left.\mathrm{S}_{8}\right)$. Este número é igual ao número de chaves de interconexão. Portanto, uma configuração radial foi obtida e o processo de otimização segue para a Etapa-7.

Etapa-7:

A Etapa-7 (etapa de substituição) tem como objetivo a realização de substituições na configuração radial obtida. Para cada chave aberta, são analisadas 2 substituições, sendo uma para cada chave adjacente com a primeira. Neste caso, o total de substituições é de 6, como mostra a Tabela 5.

Tabela 5: Substituições, sistema de 16 barras.

\begin{tabular}{|c|c|}
\hline $\begin{array}{c}\text { Chave } \\
\text { Aberta }\end{array}$ & Substituições \\
\hline $\mathrm{S}_{7}$ & $\begin{array}{c}\text { Fechamento de } \mathrm{S}_{7} \text { e abertura de } \mathrm{S}_{5} \\
\text { Fechamento de } \mathrm{S}_{7} \text { e abertura de } \mathrm{S}_{15}\end{array}$ \\
\hline $\mathrm{S}_{8}$ & $\begin{array}{c}\text { Fechamento de } \mathrm{S}_{8} \text { e abertura de } \mathrm{S}_{6} \\
\text { Fechamento de } \mathrm{S}_{8} \text { e abertura de } \mathrm{S}_{14}\end{array}$ \\
\hline $\mathrm{S}_{16}$ & $\begin{array}{c}\text { Fechamento de } \mathrm{S}_{16} \text { e abertura de } \mathrm{S}_{4} \\
\text { Fechamento de } \mathrm{S}_{16} \text { e abertura de } \mathrm{S}_{13}\end{array}$ \\
\hline
\end{tabular}

O número reduzido de substituições é fundamentado no índice de sensibilidade $S S$, Equação (3), que indica: (i) a chave ótima para abertura ou; (ii) uma "chave vizinha" adjacente da chave ótima. Esta indicação é obtida através da sensibilidade extraída do FPO pelos multiplicadores de Lagrange $\left(\lambda_{p}\right)$.

Neste caso, a configuração radial obtida após a etapa de substituição corresponde à mesma topologia indicada antes de sua realização. Ou seja, esta etapa não conduziu a uma redução adicional de perdas em relação à configuração radial obtida nas etapas anteriores. Este fato demonstra o potencial de utilização do índice de sensibilidade proposto $(S S)$, tendo em vista que neste caso as chaves ótimas para abertura foram corretamente indicadas.

$\mathrm{Na}$ topologia final obtida, as perdas neste sistema de 16 barras são de 466,13 kW. Em relação à topologia inicial, ocorreu uma redução de $8,86 \%$ no valor das perdas, como mostra a Tabela 6.

Tabela 6: Solução ARSD, sistema 16 barras.

\begin{tabular}{|c|c|c|c|}
\hline Topologia & $\begin{array}{c}\text { Perdas } \\
(\mathrm{kW})\end{array}$ & $\begin{array}{c}\text { Redução } \\
(\%)\end{array}$ & $\begin{array}{c}\text { Chaves } \\
\text { Abertas }\end{array}$ \\
\hline Inicial & 511,44 & - & $\mathrm{S}_{14}-\mathrm{S}_{15}-\mathrm{S}_{16}$ \\
\hline ARSD Proposto & $\mathbf{4 6 6 , 1 3}$ & 8,86 & $\mathrm{~S}_{16}-\mathrm{S}_{7}-\mathrm{S}_{8}$ \\
\hline
\end{tabular}

Destaca-se que a solução final obtida pelo algoritmo proposto
(ARSD) é a mesma encontrada na literatura (Civanlar $e t$. al., 1988; Gomes et. al., 2005a; Lin and Chin, 1998; Raju and Bijwe, 2008), através da utilização de outros métodos. No entanto, o ARSD oferece como vantagem eficiência computacional, tendo em vista o número reduzido de simulações de FPO necessárias para a obtenção da solução ótima.

Para comprovar esta vantagem, a Tabela 7 apresenta um quadro comparativo com o número de simulações de FPO via ARSD, necessário para a solução ótima do sistema de 16 barras, e o número de FPO e/ou FPC (Fluxo de Potência Convencional) obtido por outros métodos encontrados na literatura para este sistema.

Tabela 7: Número de simulações, sistema 16 barras.

\begin{tabular}{|c|c|}
\hline Método & Número de FPO \\
\hline ASRD Proposto & $3 \mathrm{FPO}$ \\
\hline (Raju and Bijwe, 2008) & $7 \mathrm{FPC}$ \\
\hline (Gomes(a) et. al., 2005) & $37 \mathrm{FPC}$ \\
\hline (Gomes(b) et. al., 2005) & $18 \mathrm{FPC}+3 \mathrm{FPO}$ \\
\hline
\end{tabular}

\section{2) Sistema teste de 33 Barras}

O sistema teste de 33 barras (Baran and $\mathrm{Wu}, 1989$ ), 12,66 $\mathrm{kV}$, carga total de $3715 \mathrm{~kW}$, é composto de 5 chaves de interconexão (NA). A Figura 4 mostra a topologia inicial deste sistema, na qual as chaves NA são $S_{33}, S_{34}, S_{35}, S_{36}$ e $S_{37}$. A chave $S_{1}$ não é manobrável, pois sua abertura resultaria no isolamento de todo o sistema da subestação (SE).

Para avaliar a importância da representação de diferentes níveis de carregamento no problema de reconfiguração, serão realizadas as seguintes análises:

(i) Análise-1: Considera-se somente um nível de carga média, e minimização de perdas de potência. Esta análise é importante, pois permite comparar os resultados com outras metodologias.

(ii) Análise-2: Considera-se três níveis de carregamento associados à carga leve, média e pesada, e tem como objetivo minimizar a perda total de energia durante o período de operação considerado. Nesta análise, a curva típica de carga anual do sistema é segmentada em três níveis, que correspondem aos fatores de carregamento de 0,5 p.u. para carga leve, 1,0 p.u. para carga média e 1,60 p.u. para carga pesada. As respectivas durações das cargas são de 1000, 6760 e 1000 horas (Venkatesh and Ranjan, 2006). As tarifas de energia são de $0,06 \mathrm{US} \$ \mathrm{kWh}$ (Baran and Wu, 1989) para carga leve e média, e 0,108 US $\$ / \mathrm{kWh}$ para carga pesada de acordo com a tarifação horo-sazonal azul. 


\section{Análise-1: Somente nível de carga média}

A Tabela 8 apresenta as perdas do sistema de 33 barras, na configuração inicial mostrada na Figura 4 e na topologia ótima determinada pelo algoritmo de reconfiguração proposto (ARSD).

Neste caso, a etapa de substituição (Etapa-7) do algoritmo ARSD conduz a uma redução adicional da perda, como mostra a Tabela 8. Entretanto, a abertura das chaves indicadas pelo índice de sensibilidade proposto $(S S)$ (coluna 3 ) conduz a uma redução de perda bastante satisfatória, demonstrando o potencial de aplicação do índice $S S$ para seleção de chaves.

Tabela 8: Solução Análise-1, sistema 33 barras.

\begin{tabular}{|c|c|c|c|}
\hline Topologia & Inicial & $\begin{array}{c}\text { ARSD } \\
\text { antes da } \\
\text { Etapa-7 }\end{array}$ & $\begin{array}{c}\text { ARSD } \\
\text { após a } \\
\text { Etapa-7 }\end{array}$ \\
\hline Perdas (kW) & 202,68 & 137,59 & 136,54 \\
\hline $\begin{array}{c}\text { Redução } \\
(\%)\end{array}$ & - & 32,1 & $\mathbf{3 2 , 6}$ \\
\hline Chaves & $\begin{array}{c}\mathrm{S}_{33}, \mathrm{~S}_{34}, \\
\mathrm{~S}_{35}, \mathrm{~S}_{36}, \\
\mathrm{~S}_{37}\end{array}$ & $\begin{array}{c}\mathbf{S}_{6}, \mathbf{S}_{10}, \\
\mathrm{~S}_{14}, \mathrm{~S}_{32}, \\
\mathrm{~S}_{37}\end{array}$ & $\begin{array}{c}\mathbf{S}_{7}, \mathbf{S}_{9}, \\
\mathrm{~S}_{14}, \mathrm{~S}_{32}, \\
\mathrm{~S}_{37}\end{array}$ \\
\hline
\end{tabular}

A Tabela 9 apresenta a solução obtida pelo algoritmo proposto (ARSD) e de outros métodos encontrados na literatura.

Observa-se na Tabela 9 que o algoritmo proposto para recon-

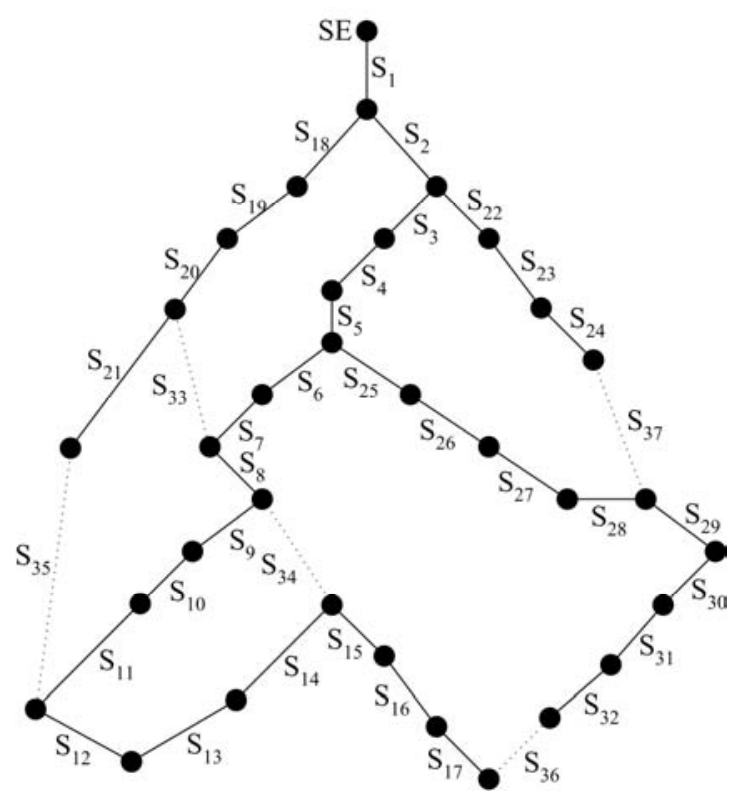

Figura 4: Sistema teste de 33 barras.
Tabela 9: Soluções, sistema 33 barras.

\begin{tabular}{|c|c|c|c|}
\hline Topologia & $\begin{array}{c}\text { Perdas } \\
(\mathrm{kW})\end{array}$ & $\begin{array}{c}\text { Redução } \\
(\%)\end{array}$ & $\begin{array}{c}\text { Chaves } \\
\text { Abertas }\end{array}$ \\
\hline Inicial & 202,68 & - & $\begin{array}{c}\mathrm{S}_{33}, \mathrm{~S}_{34}, \\
\mathrm{~S}_{35}, \mathrm{~S}_{36}, \mathrm{~S}_{37}\end{array}$ \\
\hline ARSD Proposto & 136,54 & 32,63 & $\begin{array}{c}\mathrm{S}_{7}, \mathrm{~S}_{9}, \mathrm{~S}_{14}, \\
\mathrm{~S}_{32}, \mathrm{~S}_{37}\end{array}$ \\
\hline $\begin{array}{c}\text { (Goswami and } \\
\text { Basu, 1992) }\end{array}$ & 136,54 & 32,63 & $\begin{array}{c}\mathrm{S}_{7}, \mathrm{~S}_{9}, \mathrm{~S}_{14}, \\
\mathrm{~S}_{32}, \mathrm{~S}_{37}\end{array}$ \\
\hline $\begin{array}{c}\text { (Mcdermott } \text { et } \\
\text { al., 1999) }\end{array}$ & 136,54 & 32,63 & $\begin{array}{c}\mathrm{S}_{7}, \mathrm{~S}_{9}, \mathrm{~S}_{14}, \\
\mathrm{~S}_{32}, \mathrm{~S}_{37}\end{array}$ \\
\hline $\begin{array}{c}\text { (Gomes } \text { et al., } \\
\text { 2005a) }\end{array}$ & 136,54 & 32,63 & $\begin{array}{c}\mathrm{S}_{7}, \mathrm{~S}_{9}, \mathrm{~S}_{14}, \\
\mathrm{~S}_{32}, \mathrm{~S}_{37}\end{array}$ \\
\hline $\begin{array}{c}\text { (Shirmohammadi } \\
\text { and Hong, 1989) }\end{array}$ & 136,66 & 32,57 & $\begin{array}{c}\mathrm{S}_{7}, \mathrm{~S}_{10}, \mathrm{~S}_{14}, \\
\mathrm{~S}_{32}, \mathrm{~S}_{37}\end{array}$ \\
\hline $\begin{array}{c}\text { (Gomes } \text { et al. }, \\
\text { 2005b) }\end{array}$ & 136,66 & 32,57 & $\begin{array}{c}\mathrm{S}_{7}, \mathrm{~S}_{10}, \mathrm{~S}_{14}, \\
\mathrm{~S}_{32}, \mathrm{~S}_{37}\end{array}$ \\
\hline
\end{tabular}

figuração (ARSD) apresenta resultados compatíveis com os resultados dos outros métodos.

\section{Análise-2: Três níveis de carga (leve, média e pesada)}

A Tabela 10 apresenta as perdas do sistema de 33 barras, operando na topologia inicial da Figura 4, nas condições de carregamento e tarifação definidas para a Análise-2.

Tabela 10: Solução inicial, sistema 33 barras.

\begin{tabular}{|c|c|c|c|}
\hline Carregamento & Leve & Médio & Pesado \\
\hline $\begin{array}{c}\text { Perdas por Período } \\
\text { (MWh) }\end{array}$ & 47,1 & $1.369,8$ & 575,2 \\
\hline Perda Total (MWh) & \multicolumn{3}{|c|}{$1.992,1$} \\
\hline Chaves Abertas & \multicolumn{3}{|c|}{$\mathrm{S}_{33}, \mathrm{~S}_{34}, \mathrm{~S}_{35}, \mathrm{~S}_{36}, \mathrm{~S}_{37}$} \\
\hline
\end{tabular}

A aplicação do ARSD ao sistema de 33 barras com três níveis de carregamento proporciona uma redução percentual da perda total de energia de 33,01\%, como mostra a Tabela 11 .

A Tabela 12 apresenta as configurações obtidas considerando-se cada nível de carregamento separadamente (Análise-1). Esta tabela também apresenta a configuração ótima determinada na Análise-2, que considera os três períodos de carregamento de forma simultânea.

Destaca-se que a configuração obtida na Análise-2 otimiza a operação do sistema no período de carga pesada, conforme resultado da Análise-1 (carga pesada). Porém, a configu- 
Tabela 11: Solução Análise-2, sistema 33 barras.

\begin{tabular}{|c|c|c|c|}
\hline Carregamento & Leve & Médio & Pesado \\
\hline $\begin{array}{c}\text { Perdas por Período } \\
\text { (MWh) }\end{array}$ & 32,7 & 927,9 & 373,8 \\
\hline Perda Total (MWh) & \multicolumn{3}{|c|}{$1.334,4$} \\
\hline Redução (\%) & \multicolumn{3}{|c|}{$\mathbf{3 3 , 0 1}$} \\
\hline Chaves Abertas & \multicolumn{3}{|c|}{$\mathrm{S}_{6}, \mathrm{~S}_{9}, \mathrm{~S}_{14}, \mathrm{~S}_{32}, \mathrm{~S}_{37}$} \\
\hline
\end{tabular}

Tabela 12: Configurações ótimas, sistema 33 barras.

\begin{tabular}{|c|c|}
\hline & Chaves Abertas \\
\hline $\begin{array}{c}\text { Análise-1: } \\
\text { carga leve }\end{array}$ & $\mathbf{S}_{7}, \mathrm{~S}_{9}, \mathrm{~S}_{14}, \mathrm{~S}_{32}, \mathrm{~S}_{37}$ \\
\hline $\begin{array}{c}\text { Análise-1: } \\
\text { carga média }\end{array}$ & $\mathbf{S}_{7}, \mathrm{~S}_{9}, \mathrm{~S}_{14}, \mathrm{~S}_{32}, \mathrm{~S}_{37}$ \\
\hline $\begin{array}{c}\text { Análise-1: } \\
\text { carga pesada }\end{array}$ & $\mathbf{S}_{6}, \mathrm{~S}_{9}, \mathrm{~S}_{14}, \mathrm{~S}_{32}, \mathrm{~S}_{37}$ \\
\hline $\begin{array}{c}\text { Análise-2: } \\
\text { cargas leve, média e pesada }\end{array}$ & $\mathbf{S}_{6}, \mathrm{~S}_{9}, \mathrm{~S}_{14}, \mathrm{~S}_{32}, \mathrm{~S}_{37}$ \\
\hline
\end{tabular}

ração ótima nos períodos de carga leve e média é diferente da configuração obtida na Análise-2 (troca da chave $S_{6}$ pela chave $S_{7}$ ). Entretanto, a configuração da Análise-2 minimiza a perda total considerando-se todo o período de operação incluindo os três períodos de carregamento representados. Este aspecto, mesmo para um sistema pequeno, mostra a importância da representação simultanea de diferentes níveis de carga na reconfiguração de SDE.

\section{3) Sistema Taiwan 83 barras}

O sistema de distribuição de 83 barras da Taiwan Power Corporation (TPC) (Chiou et. al., 2005) consiste de 11 alimentadores de 11,4 kV, 2 subestações e 96 circuitos. A Figura 5 mostra o diagrama deste sistema, que tem carga total de $28.350 \mathrm{~kW}$ e $20.700 \mathrm{kVAr}$. Os circuitos representados por linhas tracejadas correspondem às 13 chaves de interconexão (NA) a seguir: $\mathrm{S}_{84}, \mathrm{~S}_{85}, \mathrm{~S}_{86}, \mathrm{~S}_{87}, \mathrm{~S}_{88}, \mathrm{~S}_{89}, \mathrm{~S}_{90}, \mathrm{~S}_{91}, \mathrm{~S}_{92}$, $\mathrm{S}_{93}, \mathrm{~S}_{94}, \mathrm{~S}_{95}, \mathrm{~S}_{96}$. Estes dispositivos de chaveamento juntamente com as chaves de seccionamento (NF) totalizam 89 circuitos manobráveis. O conjunto manobrável NF é composto somente pelas chaves cuja abertura não causa ilhamento.

Neste caso, a análise é baseada em curvas típicas de carga de um sistema real de distribuição brasileiro (ANEEL, 2008). Para o Grupo-1 de alimentadores (alimentadores A-F saindo da subestação S/S1), utilizou-se a curva de carga mostrada

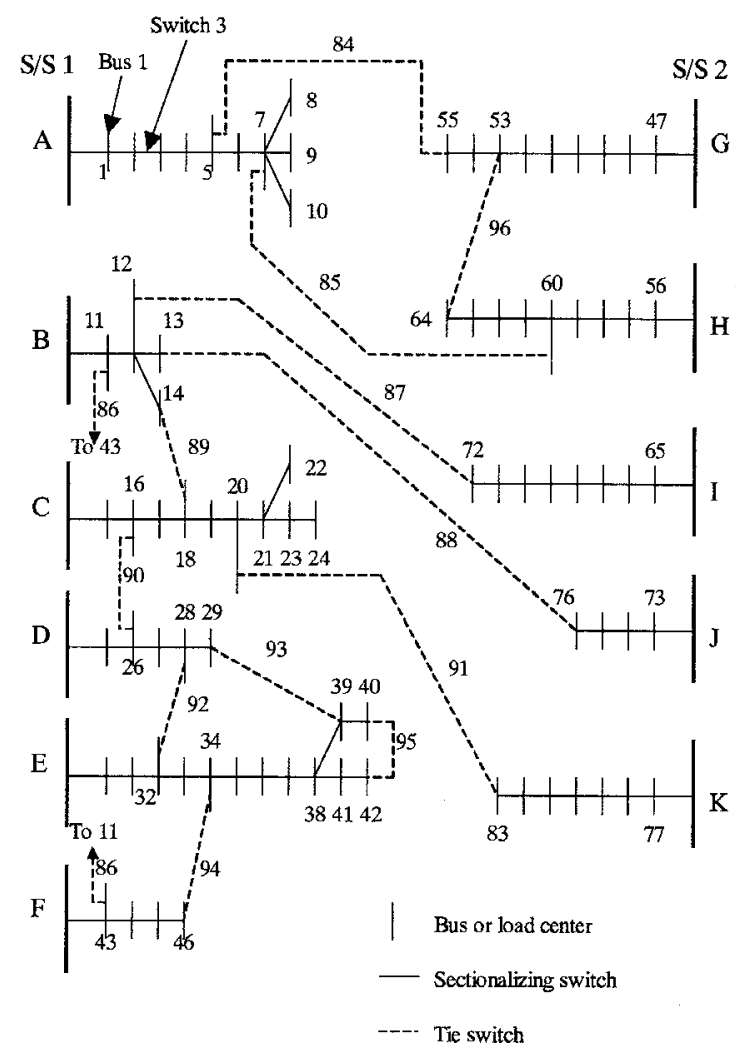

Figura 5: Sistema teste de 83 barras.

na Figura 6. A curva de carga da Figura 7 foi utilizada para o Grupo-2 de alimentadores (alimentadores G-K a partir de $\mathrm{S} / \mathrm{S} 2$ ). Estas curvas foram segmentadas em quatro patamares de carga (N1-N4), os quais são descritos na Tabela 13.

Destaca-se que a duração em horas está associada ao período anual de operação do sistema, correspondente ao período diário de cada nível de carga conforme as Figuras 6 e 7. Neste caso, o nível N3 corresponde ao horário de ponta e, portanto, sua tarifa é maior que a dos demais níveis, conforme tarifação horo-sazonal azul.

A Tabela 14 apresenta as perdas deste sistema de 83 barras para: (i) a topologia inicial; (ii) a topologia encontrada em (Chiou et. al., 2005); (iii) a topologia proposta através do algoritmo ARSD considerando-se os quatro níveis de carregamento simultaneamente nos dois alimentadores. O período de operação considerado é de um ano, e as condições de carregamento e tarifação são aquelas descritas para o Grupo-1 e o Grupo-2.

Verifica-se, portanto, que a reconfiguração deste sistema de distribuição considerando-se os quatro níveis de carga (N1N4) implica em uma redução de perdas no Grupo-1, e um aumento de perdas no Grupo-2. Isto ocorre porque a recon- 


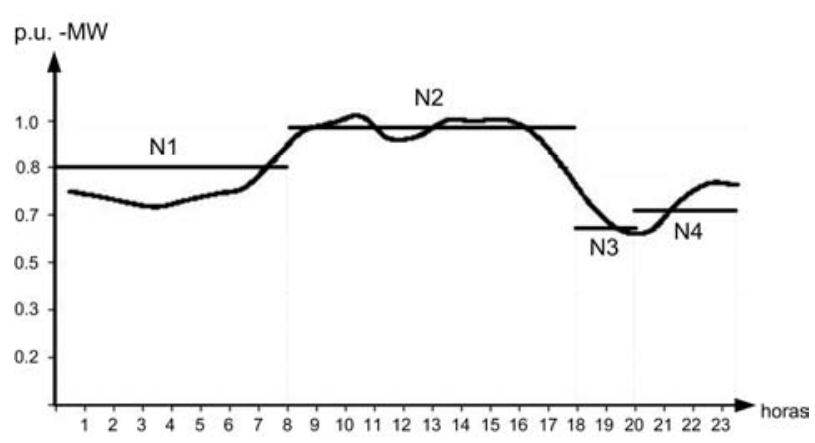

Figura 6: Curva típica de carga do Grupo-1.

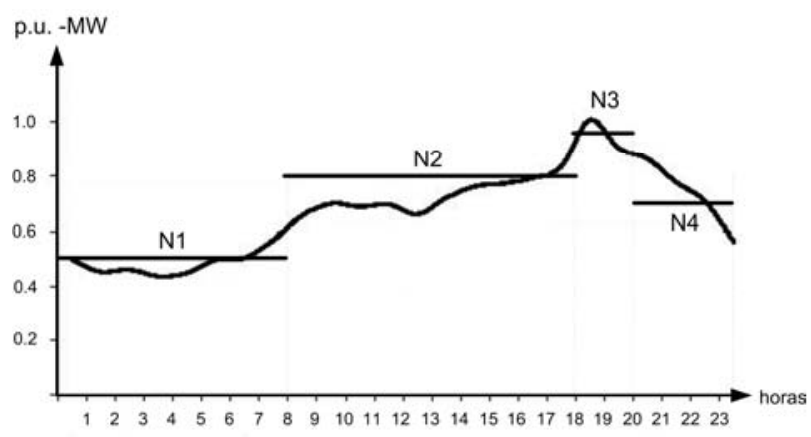

Figura 7: Curva típica de carga do Grupo-2.

figuração conduz a uma transferência de carga do Grupo-1 para o Grupo-2. Entretanto, a perda total nos dois alimentadores é reduzida.

Destaca-se que a configuração ótima obtida para os quatro períodos de carregamento do sistema é diferente da configuração obtida por (Chiou et. al., 2005), que consideram somente um nível de carga. Esta diferença justifica a importância da representação dos diferentes níveis de carga para a reconfiguração de SDE.

A Tabela 15 apresenta os tempos computacionais de execução do ARSD para cada um dos três sistemas testados. Es-

Tabela 13: Segmentação das curvas sistema 83 barras.

\begin{tabular}{|c|c|c|c|c|}
\hline Nível & $\mathrm{N} 1$ & $\mathrm{~N} 2$ & $\mathrm{~N} 3$ & $\mathrm{~N} 4$ \\
\hline $\begin{array}{c}\text { Fator de Carga (p.u.) } \\
\text { Grupo-1 }\end{array}$ & 0,80 & 0,95 & 0,60 & 0,70 \\
\hline $\begin{array}{c}\text { Fator de Carga (p.u.) } \\
\text { Grupo-2 }\end{array}$ & 0,50 & 0,80 & 0,95 & 0,70 \\
\hline Duração (horas) & 2.920 & 3.650 & 730 & 1.460 \\
\hline $\begin{array}{c}\text { Tarifa de Energia } \\
\text { (US\$/kWh) }\end{array}$ & 0,06 & 0,06 & 0,108 & 0,06 \\
\hline
\end{tabular}

Tabela 14: Soluções, sistema 83 barras.

\begin{tabular}{|c|c|c|c|}
\hline Configuração & Inicial & $\begin{array}{l}\text { (Chiou et. } \\
\text { al., 2005) }\end{array}$ & $\begin{array}{c}\text { Proposta } \\
\text { (ARSD) }\end{array}$ \\
\hline $\begin{array}{l}\text { Chaves } \\
\text { Abertas }\end{array}$ & $\begin{array}{c}\mathrm{S}_{84}, \mathrm{~S}_{85}, \\
\mathrm{~S}_{86}, \mathrm{~S}_{87}, \\
\mathrm{~S}_{88}, \mathrm{~S}_{89}, \\
\mathrm{~S}_{90}, \mathrm{~S}_{91}, \\
\mathrm{~S}_{92}, \mathrm{~S}_{93}, \\
\mathrm{~S}_{94}, \mathrm{~S}_{95}, \\
\quad \mathrm{~S}_{96}\end{array}$ & $\begin{array}{c}\mathrm{S}_{7}, \mathrm{~S}_{13}, \\
\mathrm{~S}_{34}, \mathrm{~S}_{39} \\
\mathrm{~S}_{41}, \mathbf{S}_{55} \\
\mathrm{~S}_{62}, \mathrm{~S}_{72}, \\
\mathbf{S}_{83}, \mathrm{~S}_{86} \\
\mathrm{~S}_{89}, \mathrm{~S}_{90} \\
\quad \mathrm{~S}_{92}\end{array}$ & $\begin{array}{c}\mathrm{S}_{7}, \mathrm{~S}_{13}, \\
\mathrm{~S}_{34}, \mathrm{~S}_{39}, \\
\mathrm{~S}_{42}, \mathbf{S}_{84}, \\
\mathrm{~S}_{62}, \mathrm{~S}_{72}, \\
\mathbf{S}_{91}, \mathrm{~S}_{86}, \\
\mathrm{~S}_{89}, \mathrm{~S}_{90}, \\
\quad \mathrm{~S}_{92}\end{array}$ \\
\hline $\begin{array}{c}\text { Perdas } \\
\text { Grupo-1 } \\
(\mathrm{MWh})\end{array}$ & $1.354,6$ & 963,4 & 862,4 \\
\hline $\begin{array}{c}\text { Perdas } \\
\text { Grupo-2 } \\
(\mathrm{MWh})\end{array}$ & $1.497,1$ & $1.497,5$ & $1.587,8$ \\
\hline $\begin{array}{l}\text { Perdas Totais } \\
\text { (MWh) }\end{array}$ & $2.851,7$ & $2.460,9$ & $2.450,2$ \\
\hline
\end{tabular}

tes valores correspondem às médias dos tempos de processamento calculadas após dez execuções do ARSD para cada sistema. Esta tabela também apresenta os números de simulações do problema de FPO formulado em (2).

Tabela 15: Tempo computacional médio ARSD.

\begin{tabular}{|c|c|c|}
\hline Sistema & $\begin{array}{c}\text { Tempo } \\
\text { (segundos) }\end{array}$ & Número de FPO \\
\hline 16 Barras & 1,56 & 3 \\
\hline $\begin{array}{c}33 \text { Barras, } \\
\text { Análise-1 }\end{array}$ & 3,72 & 15 \\
\hline $\begin{array}{c}\text { 33 Barras, } \\
\text { Análise-2 }\end{array}$ & 11,81 & 15 \\
\hline Taiwan 83 Barras & 77,71 & 39 \\
\hline
\end{tabular}

Tendo em vista que o ARSD trata-se de um algoritmo heurístico construtivo, os tempos computacionais envolvidos na sua execução são pequenos, como se pode observar na coluna 2 da Tabela 15. A coluna 3 apresenta o número de simulações do FPO formulado em (2) para a obtenção da solução ótima de cada sistema testado. O número de simulações de FPO e/ou FPC não foi encontrado nas referências utilizadas para comparação dos resultados dos sistemas de 33 e 83 barras.

As linhas 3 e 4 da Tabela 15 apresentam os tempos para o sistema de 33 barras, obtidos respectivamente para o nível de carga média (Análise-1), e para os níveis de carga leve, média e pesada considerados simultaneamente (Análise-2). 
Observa-se que a inclusão dos níveis de carregamento na análise não introduz aumento substancial do tempo de processamento. Portanto, a aplicação da metodologia proposta permite a inclusão de aspectos importantes, mantendo-se os tempos computacionais dentro de uma faixa viável para aplicação em tempo-real. Além disto, a implementação desta metodologia em uma plataforma computacional $\mathrm{C}++$ poderia implicar em menores tempos de processamento, favorecendo a aplicação do ARSD para sistemas de grande porte, também em tempo-real.

\section{CONCLUSÃO}

Este trabalho apresentou um algoritmo de otimização para a reconfiguração de sistemas de distribuição, com o objetivo de minimização da perda total de energia durante períodos com diferentes níveis de carregamento. Considerando-se as elevadas dimensões deste problema, esta tarefa é complexa uma vez que o número de alternativas é elevado devido a sua natureza combinatória. Através dos resultados obtidos, os seguintes pontos podem ser destacados:

- O método proposto apresentou como vantagem a solução do problema com pequeno esforço computacional devido ao reduzido número de execuções de FPO, favorecendo a aplicação do mesmo em sistemas de grande porte considerando-se mais de um nível de carregamento.

- A qualidade das configurações obtidas para os sistemas simulados mostrou-se compatível àquelas encontradas na literatura.

- O método considera as características de radialidade e conectividade.

- A metodologia apresenta a exploração do potencial de aplicação dos multiplicadores de Lagrange como índices de sensibilidade para definição de configurações ótimas.

\section{AGRADECIMENTOS}

Os autores gostariam de agradecer a CAPES, CNPq e FAPEMIG.

\section{REFERÊNCIAS}

Sarfi, R. J.; Salama, M. M. A. and Chikhani, A. Y. (1994). A survey of the state of the art in distribution system reconfiguration for system loss reduction. Electric Power Systems Res., Vol. XXXI, No. 1, pp. 61-70.
Kalantar, M.; Dashti, R. and Dashti, R. (2006). Combination of network reconfiguration and capacitor placement for loss reduction in distribution system with based genetic algorithm, Proc. of the 41st International Universities Power Engineering Conf. (UPEC '06), Newcastle upon Tyne, UK, Vol. I, pp. 308-312.

Merlin A. and Back, G. (1975). Search for minimum-loss operational spanning tree configuration for urban power distribution system, Proc. 5th Power System Conf., Cambridge, pp. 1-18.

Liu, C. C.; Lee, S. J. and Vu, K. (1989). Loss minimization of distribution feeders: optimality and algorithms. IEEE Trans. Power Delivery, Vol. IV, No. 2, pp. 1281-1289.

Radha, B.; Ah King, R. T. F. and Rughooputh, H. C. S. (2003). Optimal network reconfiguration of electrical distribution systems. IEEE Proc. International Conf. on Industrial Technology, Vol. I, pp. 66-71.

Chiang, H. and Jean-Jumeau, R. (1990). Optimal network reconfiguration in distribution system: part 2: solution algorithms and numerical results. IEEE Trans. Power Delivery, Vol. V, pp. 1568-1574.

Khoa, T. Q. D. and Phan, B. T. T. (2006). Ant colony searchbased loss minimum for reconfiguration of distribution systems, IEEE Power India Conference, New Delhi, pp. 6.

Mori, H. and Ogita, Y. (2000). A parallel tabu search based method for reconfigurations of distribution systems, IEEE Summer Power Engineering Society Meeting, Seattle, WA, USA, Vol. I, pp. 73-78.

Nara, K.; Shiose, A.; Kitagawa, M. and Ishihara, T. (1992). Implementation of genetic algorithm for distribution systems loss minimum re-configuration. IEEE Trans. Power Systems, Vol. VII, No. 3, pp. 1044-1051.

Salazar, H.; Gallego, R. and Romero, R. (2006). Artificial neural networks and clustering techniques applied in the reconfiguration of distribution systems. IEEE Trans. Power Delivery, Vol. XXI, No. 3, pp. 1735-1742.

Das, D. (2006). A fuzzy multiobjective approach for network reconfiguration of distribution systems. IEEE Trans. Power Delivery, Vol. XXI, No. 1, pp. 202-209.

Shirmohammadi, D. and Hong, H. W. (1989). Reconfiguration of electric distribution for resistive line loss reduction. IEEE Trans. Power Delivery, Vol. IV, No. 2, pp. 1492-1498.

Goswami, S. K. and Basu, S. K. (1992). A new algorithm for the reconfiguration of distribution feeders for loss minimization. IEEE Trans. Power Delivery, Vol. VII, No. 3, pp. 1484-1491. 
Peponis, G. and Papadopoulos M. (1995). Reconfiguration of radial distribution networks: application of heuristic methods on large-scale networks. IEE Proceedings Generation, Transmission and Distribution, Vol. CXLII, No. 6, pp. 631-638.

Kagan, N. and Oliveira, C. C. B. (1998). Reconfiguração de redes de distribuição de energia elétrica através de ferramenta para solução de problemas de decisão com múltiplos objetivos e incertezas. SBA Controle e Automação, Vol. IX, No. 1, pp. 18-30.

McDermott, T. E.; Drezga, I. and Broadwater, R. P. (1999). A heuristic nonlinear constructive method for distribution system reconfiguration. IEEE Trans. Power Systems, Vol. XIV, No. 2, pp. 478-483.

Civanlar, S.; Grainger, J. J.; Yin, H. and Lee, S. S. H. (1988). Distribution feeder reconfiguration for loss reduction. IEEE Trans. Power Delivery, Vol. III, No. 3, pp. 12171223.

Baran, M. E. and Wu, F. F. (1989). Network reconfiguration in distribution systems for loss reduction and load balancing. IEEE Trans. Power Delivery, Vol. IV, No. 2, pp. 1401-1407.

Shin, J. R.; Kim, B. S.; Park, J. B. and Lee, K. Y. (2007). A new optimal routing algorithm for loss minimization and voltage stability improvement in radial power system. IEEE Trans. Power Systems, Vol. XXII, No. 2, pp. 648-657.

Mantovani, J. R. S.; Casari, F. and Romero, R. A. (2000). Reconfiguração de sistemas de distribuição radiais utilizando o critério de queda de tensão. SBA Controle $e$ Automação, Vol. XI, No. 3, pp. 150-159.

Gomes, F. V.; Carneiro, S.; Pereira, J. L. R.; Vinagre, M. P. and Garcia, P. A. N. (2005a). A new heuristic reconfiguration algorithm for large distribution systems. IEEE Trans. Power Systems, Vol. XX, No. 3, pp. 1373-1378.

Gomes, F. V.; Carneiro, S.; Pereira, J. L. R.; Vinagre, M. P.; Garcia, P. A. N.; Oliveira, E. J. and Araújo, L. R. (2005b). A new distribution system reconfiguration approach using optimal power flow technique and sensitivity analysis for loss reduction, IEEE Power Engineering Society General Meeting, Vol. I, No. 1, pp. 1-5.

Chen, S. and Cho, M. Y. (1993). Energy loss reduction by critical switches. IEEE Trans. Power Delivery, Vol. VIII, No. 3, pp. 1246-1253.

Taleski, R. and Rajicic, D. (1997). Distribution network reconfiguration for energy loss reduction. IEEE Trans. Power Systems, Vol. XII, No. 1, pp. 398-406.
Dumbrava, V.; Comanescu, G. and Coculescu, S. (2001). Reconfiguration of the operation diagrams of urban electricity distribution networks by minimizing the energy losses, 16th International Conf. and Exhibition on Electricity Distribution, Part I: Contributions, CIRED, (IEE Conf. Publ. No. 482), Amsterdam, Vol. V, pp. 5.

Venkatesh, B. and Ranjan, R. (2006). Fuzzy EP algorithm and dynamic data structure for optimal capacitor allocation in radial distribution systems. IEE Proceedings Generation, Transmission and Distribution, Vol. CLIII, No. 1, pp. 80-88.

Silva Junior, I. C.; Carneiro Junior, S.; Oliveira, E. J.; Costa, J. S.; Pereira, J. L. R. and Garcia, P. A. N. (2008). A heuristic constructive algorithm for capacitor placement on distribution systems. IEEE Trans. Power Systems, Vol. XXIII, No. 4, pp. 1619-1626.

Karmakar, N. K. (1991). Computational Results of an Interior Point Algorithm for Large Scale Linear Programming, Mathematical Programming, 52, pp. 555-586.

Granville, S.; Melo, J. C. O. and Melo, A. C. G. (1996). Application of interior point methods to power flow unsolvability. IEEE Trans. Power Systems, Vol. XI, No. 2, pp. 1096-1103.

Haykin, S. (2002). Neural Networks: A Comprehensive Foundation, Ontario, Prentice Hall, 2nd edition.

Oliveira, E. J.; Silva Junior, I. C.; Pereira, J. L. R. and Carneiro Junior, S. (2005). Transmission system expansion planning using a sigmoid function to handle integer investment variables. IEEE Trans. Power Systems, Vol. I, No. 1, pp. 1616-1621.

Lin, W. M. and Chin, H. C. (1998). A New approach for distribution feeder reconfiguration for loss reduction and service restoration. IEEE Trans. Power Delivery, Vol. XIII, No. 3, pp. 870-875.

Goldbarg, M. C. and Luna, H. P. L. (2005). Otimização Combinatória e Programação Linear: modelos e algoritmos, Elsevier, 2nd edition.

Chiou, J. P.; Chung, C. F. and Su, C. T. (2005). Variable scaling hybrid differential evolution for solving network reconfiguration of distribution systems. IEEE Trans. Power Systems, Vol. XX, No. 2, pp. 668-674.

Raju, G. K. V. and Bijwe, P. R. (2008). An efficient algorithm for loss reconfiguration of distribution system based on sensitivity and heuristics. IEEE Trans. Power Systems, Vol. XXIII, No. 3, pp. 1280-1287. 
Agência Nacional de Energia Elétrica (Brasil). Superintendência de Regulação dos Serviços de Distribuição. Nota técnica $n^{\circ}$ 0076/2008 SRD/ANEEL. Brasília, 2008. Disponível em: www.aneel.gov.br. Acesso em: 25 ago. 2008. 